Proceedings of the 17th Czech and Slovak Conference on Magnetism, Košice, Slovakia, June 3-7, 2019

\title{
Thermodynamic Properties of Superconducting State in Intercalated Bilayer Graphene
}

\author{
K.M. SkOCZYLAS ${ }^{a, *}$, A.P. DURAJSKI ${ }^{b}$ AND R. SzCZȨŚNIAK ${ }^{b}$ \\ ${ }^{a}$ Institute of Physics, Jan Długosz University in Czȩstochowa, \\ al. Armii Krajowej 13/15, 42-200 Częstochowa, Poland
}

${ }^{b}$ Institute of Physics, Czȩstochowa University of Technology, al. Armii Krajowej 19, 42-200 Czȩstochowa, Poland

\begin{abstract}
To this day, no evidence of superconducting state in monolayer graphene has been observed. However, the easily manageable two dimensional structure of graphene through almost freely doping allows to change the properties of the structure. In fact, the resistivity drop has been detected in graphene bilayer tilted by small angle and Ca intercalated bilayer. This work comprises investigation on the thermodynamic properties of the superconducting state in intercalated graphene bilayer system $\mathrm{C}_{6} \mathrm{XC}_{6}$ (where $\mathrm{X}$ is either $\mathrm{K}, \mathrm{Ca}, \mathrm{Rb}$ or $\mathrm{Sr}$ ). Eliashberg theory of superconductivity allowed to compute on the quantitative level the thermodynamic quantities, including the transition temperature, free energy, entropy and specific heat difference between the superconducting and the normal state. Highest critical temperature was obtained for calcium intercalated bilayer $\left(T_{c}=14.56 \mathrm{~K}\right)$, while the insertion of potassium and strontium lead to similar temperature values of $8.67 \mathrm{~K}$ and $8.74 \mathrm{~K}$. In addition, the calculated BCS ratios: $R_{\Delta}, R_{C}$, and $R_{H}$ deviate significantly from the constant, theoretical values.
\end{abstract}

DOI: 10.12693/APhysPolA.137.776

PACS/topics: Superconductivity, graphene, bilayer

\section{Introduction}

Studies show superior physical and electric properties of carbon in atomic layer form which are the main stimulus to investigate other possible features of graphene in other areas of research. Unfortunately, superconducting state is out of reach for pristine, monolayer graphene. Notwithstanding, two dimensional structure allow for doping in many ways and having this in mind, electrical resistance drop was found in $\mathrm{Li}$ decorated monolayer and $\mathrm{Ca}$ intercalated graphene bilayer [1, 2]. Recent work of the research group of Cao et al. [3] proved the existence of superconducting state in pristine, twisted graphene bilayer. Furthermore, theoretical investigations with the use of density functional theory predicted $\mathrm{C}_{6} \mathrm{CaC}_{6}$ structure to have the transition temperature $\left(T_{c}\right)$ in the range between $6.8 \mathrm{~K}$ and $8.1 \mathrm{~K}$ [4].
Considering the above observations, our group had done the study on electron-phonon structure of graphene bilayer intercalated with the I and II group elements, namely: K, Ca, Rb, Sr [5]. Results of the above work stands as a basis for this research, where using the strongcoupling Eliashberg theory of superconductivity [6], we investigate the thermodynamic properties of the superconducting state in $\mathrm{C}_{6} \mathrm{KC}_{6}, \mathrm{C}_{6} \mathrm{CaC}_{6}, \mathrm{C}_{6} \mathrm{RbC}_{6}$, and $\mathrm{C}_{6} \mathrm{SrC}_{6}$ systems.

\section{Theoretical model}

The Eliashberg formalism provides a description of the order parameter $\phi(\omega)$ and the wave function renormalization factor $Z(\omega)$ in the mixed representation on the imaginary and real frequency axis, basing on the Nambu formalism and the Green's function theory [7-9]. The Eliashberg formulas can be written as

$$
\begin{aligned}
& \phi(\omega)=\frac{\pi}{\beta} \sum_{m=-M}^{M} \frac{\lambda\left(\omega-\mathrm{i} \omega_{m}\right)-\mu^{\star} \theta\left(\omega_{c}-\left|\omega_{m}\right|\right)}{\sqrt{\omega_{m}^{2} Z_{m}^{2}+\phi_{m}^{2}}} \phi_{m} \\
& +\mathrm{i} \pi \int_{0}^{+\infty} \mathrm{d} \omega^{\prime} \alpha^{2} F\left(\omega^{\prime}\right)\left[\left(N\left(\omega^{\prime}\right)+f\left(\omega^{\prime}-\omega\right)\right) K\left(\omega,-\omega^{\prime}\right) \phi\left(\omega-\omega^{\prime}\right)\right] \\
& +\mathrm{i} \pi \int_{0}^{+\infty} \mathrm{d} \omega^{\prime} \alpha^{2} F\left(\omega^{\prime}\right)\left[\left(N\left(\omega^{\prime}\right)+f\left(\omega^{\prime}+\omega\right)\right) K\left(\omega, \omega^{\prime}\right) \phi\left(\omega+\omega^{\prime}\right)\right],
\end{aligned}
$$

*corresponding author; e-mail: kamil.skoczylas@ujd.edu.pl 


$$
\begin{aligned}
& Z(\omega)=1+\frac{\mathrm{i} \pi}{\omega \beta} \sum_{m=-M}^{M} \frac{\lambda\left(\omega-\mathrm{i} \omega_{m}\right) \omega_{m}}{\sqrt{\omega_{m}^{2} Z_{m}^{2}+\phi_{m}^{2}}} Z_{m} \\
& +\frac{\mathrm{i} \pi}{\omega} \int_{0}^{+\infty} \mathrm{d} \omega^{\prime} \alpha^{2} F\left(\omega^{\prime}\right)\left[\left(N\left(\omega^{\prime}\right)+f\left(\omega^{\prime}-\omega\right)\right) K\left(\omega,-\omega^{\prime}\right)\left(\omega-\omega^{\prime}\right) Z\left(\omega-\omega^{\prime}\right)\right] \\
& +\frac{\mathrm{i} \pi}{\omega} \int_{0}^{+\infty} \mathrm{d} \omega^{\prime} \alpha^{2} F\left(\omega^{\prime}\right)\left[\left(N\left(\omega^{\prime}\right)+f\left(\omega^{\prime}+\omega\right)\right) K\left(\omega, \omega^{\prime}\right)\left(\omega+\omega^{\prime}\right) Z\left(\omega+\omega^{\prime}\right)\right]
\end{aligned}
$$

$$
K\left(\omega, \omega^{\prime}\right)=\frac{1}{\sqrt{\left(\omega+\omega^{\prime}\right)^{2} Z^{2}\left(\omega+\omega^{\prime}\right)-\phi^{2}\left(\omega+\omega^{\prime}\right)}} .
$$

Now, the complex frequency-dependent gap function can be exemplified as $\Delta(\omega)=\phi(\omega) / Z(\omega)$. On the imaginary axis the functions $\phi_{n}=\phi\left(\mathrm{i} \omega_{n}\right)$ and $Z_{n}=Z\left(\mathrm{i} \omega_{n}\right)$ can be expressed in the following form [6]:

$$
\begin{aligned}
& \phi_{m}=\frac{\pi}{\beta} \sum_{n=-M}^{M} \frac{\lambda\left(\mathrm{i} \omega_{m}-\mathrm{i} \omega_{n}\right)-\mu^{\star} \theta\left(\omega_{c}-\left|\omega_{n}\right|\right)}{\sqrt{\omega_{n}^{2} Z_{n}^{2}+\phi_{n}^{2}}} \phi_{n}, \\
& Z_{m}=1+\frac{1}{\omega_{m}} \frac{\pi}{\beta} \sum_{n=-M}^{M} \frac{\lambda\left(\mathrm{i} \omega_{m}-\mathrm{i} \omega_{n}\right)}{\sqrt{\omega_{n}^{2} Z_{n}^{2}+\phi_{n}^{2}}} \omega_{n} Z_{n}, \\
& \lambda(z)=2 \int_{0}^{\Omega_{\max }} \mathrm{d} \Omega \frac{\Omega}{\Omega^{2}-z^{2}} \alpha^{2} F(\Omega),
\end{aligned}
$$

using Matsubara frequencies defined as: $\omega_{m}=$ $(\pi / \beta)(2 m-1)$, where $m=0, \pm 1, \pm 2, \ldots, \pm M$, and $M=1100$. Furthermore, $\beta=\left(k_{\mathrm{B}} T\right)^{-1}$, where $k_{\mathrm{B}}$ denotes the Boltzmann constant, and $\lambda(z)$ is the pairing kernel. The Eliashberg spectral functions, i.e., $\alpha^{2} F(\Omega)$, are taken from our previous work on the electron-phonon structure of the $\mathrm{C}_{6} \mathrm{XC}_{6}$ group [5]. Here, for every studied doping the maximum phonon frequency $\Omega_{\max }$ is set to $198 \mathrm{meV}$, based on given $\alpha^{2} F(\Omega)$ functions, and followed by setting of the cessation frequency $\omega_{c}$ to the same value. Depairing effects are reproduced by the term: $\mu^{\star} \theta\left(\omega_{c}-\left|\omega_{n}\right|\right)$, where $\mu^{\star}$ is the Coulomb pseudopotential parameter [11] set to 0.15 .

\section{Computational results}

The order parameter $\Delta_{m=1}$ and the wave function renormalization factor function for the first Matsubara frequency $Z_{m=1}$ can be traced in Fig. 1. Non-zero values of the $\Delta_{m=1}$ function (Fig. 1a) illustrate the existence of the superconducting state within the material. It can be clearly seen that Ca-doped graphene bilayer expresses highest transition temperature. Values of critical temperatures obtained for $\mathrm{C}_{6} \mathrm{KC}_{6}, \mathrm{C}_{6} \mathrm{CaC}_{6}, \mathrm{C}_{6} \mathrm{RbC}_{6}$, and $\mathrm{C}_{6} \mathrm{SrC}_{6}$ are: $8.67 \mathrm{~K}, 14.56 \mathrm{~K}, 5.47 \mathrm{~K}$, and $8.74 \mathrm{~K}$, respectively. The wave function renormalization factor for the first Matsubara frequency (Fig. 1b) is implemented as the electron effective mass by the relation: $m_{e}^{\star}=m_{e} Z_{m=1}$, where $m_{e}$ denotes the electron band mass. Usually, higher values of $\Delta_{m=1}$ function move on to $Z_{m=1}$ function values, which does not hold in the case of Rb-doping, compared to the rest of the systems.

The free energy difference function $\Delta F$ between the superconducting $Z^{S}$ and the normal $Z^{N}$ state can be computed using the wave function renormalization factors of the $Z^{S}$ and $Z^{N}$ state [10]. Knowing the $\Delta F$ one can obtain the entropy difference $\Delta S$, and the specific heat difference $\Delta C$ between the $Z^{S}$ and $Z^{N}$ state, together with the thermodynamic critical field function $H_{c}$. Thus, the following formulas were used:

$$
\begin{aligned}
& \frac{\Delta F}{\rho(0)}=-\frac{2 \pi}{\beta} \sum_{n=1}^{M}\left(\sqrt{\omega_{n}^{2}+\Delta_{n}^{2}}-\left|\omega_{n}\right|\right) \\
& \quad \times\left(Z_{n}^{S}-Z_{n}^{N} \frac{\left|\omega_{n}\right|}{\sqrt{\omega_{n}^{2}+\Delta_{n}^{2}}}\right), \\
& \frac{H_{c}}{\sqrt{\rho(0)}}=\sqrt{-8 \pi \frac{\Delta F}{\rho(0)}} \\
& \frac{\Delta C}{k_{\mathrm{B}} \rho(0)}=-\frac{1}{\beta} \frac{\mathrm{d}^{2}(\Delta F / \rho(0))}{\mathrm{d}\left(k_{\mathrm{B}} T\right)^{2}}, \\
& \frac{\Delta S}{k_{\mathrm{B}} \rho(0)}=-\frac{\mathrm{d}(\Delta F / \rho(0))}{\mathrm{d}\left(k_{\mathrm{B}} T\right)} .
\end{aligned}
$$

Here, the $\rho(0)$ term defines the electron density of states at the Fermi level. Results for the free energy difference and the thermodynamic critical field functions can be seen in Fig. 2. In the case of $\Delta F$ function (Fig. 2b), negative values describe the thermodynamic stability of the superconducting state below the critical temperature. Similarly, the thermodynamic critical field
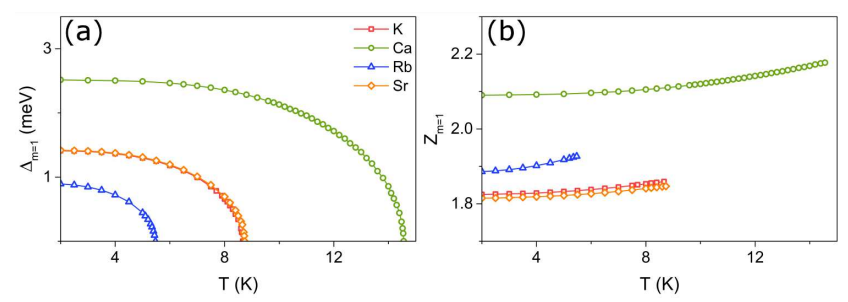

Fig. 1. Results for the order parameter (a), and (b) the wave function renormalization factor for studied dopants. 


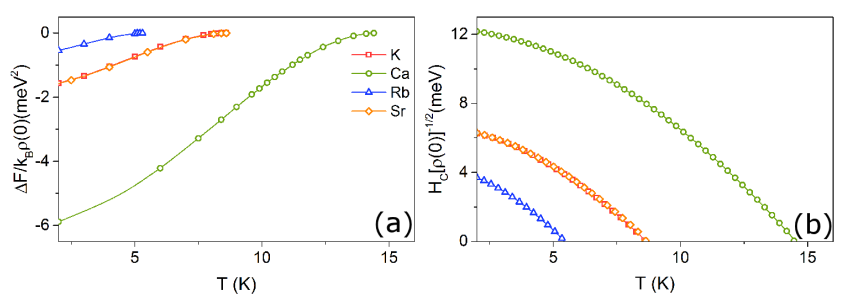

Fig. 2. Obtained functions of the free energy difference between the superconducting and the normal state (a), and (b) thermodynamic critical field.
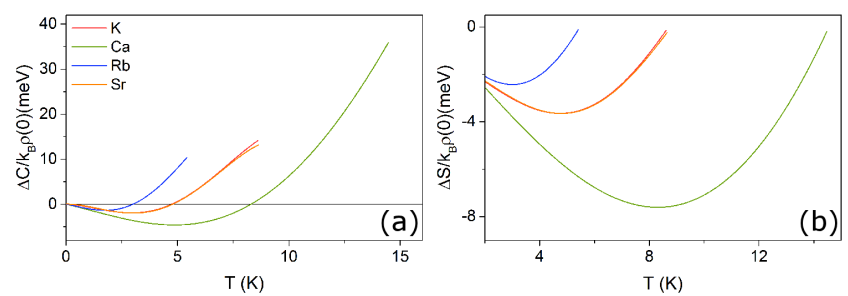

Fig. 3. The specific heat and entropy difference functions obtained for $\mathrm{C}_{6} \mathrm{KC}_{6}, \mathrm{C}_{6} \mathrm{CaC}_{6}, \mathrm{C}_{6} \mathrm{RbC}_{6}$, and $\mathrm{C}_{6} \mathrm{SrC}_{6}$ systems.

\section{TABLE I}

Results for dimensionless BCS ratios $R_{\Delta}, R_{H}$ and $R_{C}$.

\begin{tabular}{c|c|c|c|c|c}
\hline \hline & $\begin{array}{c}\text { BCS } \\
\text { theory }\end{array}$ & $\mathrm{C}_{6} \mathrm{KC}_{6}$ & $\mathrm{C}_{6} \mathrm{CaC}_{6}$ & $\mathrm{C}_{6} \mathrm{RbC}_{6}$ & $\mathrm{C}_{6} \mathrm{SrC}_{6}$ \\
\hline$R_{\Delta}$ & 3.53 & 3.80 & 4.08 & 3.86 & 3.80 \\
$R_{H}$ & 0.168 & 0.155 & 0.151 & 0.160 & 0.154 \\
$R_{C}$ & 1.43 & 1.56 & 2.01 & 1.74 & 1.45
\end{tabular}

function (Fig. 2b) depicts the durability of the superconducting state on the magnetic field and current flow through the material. Noteworthy, $\mathrm{C}_{6} \mathrm{CaC}_{6}$ system leads in both characteristics compared to the rest of studied structures.

The specific heat jump, characteristic to the transition to the superconducting state can be traced in Fig. 3a. The entropy difference function is drawn in Fig. 3b, and it reveals that electrons within the material in the superconducting state are more ordered comparing to the normal state.

With the above functions one can determine the BCS ratios [12]: $R_{\Delta}, R_{H}, R_{C}$, using the following formulas: $R_{\Delta}=2 \Delta(0) /\left(k_{\mathrm{B}} T_{c}\right), R_{H}=T_{c} C^{N}\left(T_{c}\right) / H_{c}^{2}(0)$, and $R_{C}=\Delta C\left(T_{c}\right) / C^{N}\left(T_{c}\right)$. Results for the above quantities are collated with the Bardeen-Cooper-Schrieffer constant values in Table I. Large discrepancies for every ratio can be noted, those results are strictly connected to the fact of BCS theory having not taking into consideration the strong-coupling interactions [10].

\section{Conclusions}

In this paper, the thermodynamic properties of the superconducting state of intercalated bilayer graphene $\left(\mathrm{C}_{6} \mathrm{KC}_{6}, \mathrm{C}_{6} \mathrm{CaC}_{6}, \mathrm{C}_{6} \mathrm{RbC}_{6}\right.$, and $\mathrm{C}_{6} \mathrm{SrC}_{6}$ systems $)$ are revealed. Obtained thermodynamic quantities indicate the best characteristics for Ca-intercalated structure with the transition temperature of $14.56 \mathrm{~K}$. Based on the $\Delta(0), \Delta C$, and $H_{C}$ functions, BCS ratios have been attained, which show that the Bardeen-Cooper-Schrieffer theory cannot properly describe the superconducting state within studied systems.

\section{References}

[1] B.M. Ludbrook, G. Levy, P. Nigge, M. Zonno, M. Schneider, D.J. Dvorak, C.N. Veenstra, S. Zhdanovich, D. Wong, P. Dosanjh, C. Straßer, A. Stöhr, S. Forti, C.R. Ast, U. Starke, A. Damascelli, Proc. Natl. Acad. Sci. 112, 38 (2015).

[2] J. Chapman, Y. Su, C.A. Howard, D. Kundys, A.N. Grigorenko, F. Guinea, A.K. Geim, I.V. Grigorieva, R.R. Nair, Sci. Rep. 6, 23254 (2016).

[3] Y. Cao, V. Fatemi, A. Demir, S. Fang, S.L. Tomarken, J.Y. Luo, J.D. Sanchez-Yamagishi, K. Watanabe, T. Taniguchi, E. Kaxiras, R.C. Ashoori, P. JarilloHerrero, Nature 556, 7699 (2018).

[4] E.R. Margine, H. Lambert. F. Giustino, Sci. Rep. 6, 21414 (2016).

[5] A.P. Durajski, K.M. Skoczylas, R. Szczȩśniak, Phys. Chem. Chem. Phys. 21, 5925 (2019).

[6] G.M. Eliashberg, Sov. Phys. JETP 11, 696 (1960).

[7] Y. Nambu, Phys. Rev. 117, 648 (1960).

[8] N.N. Bogolyubov, S.V. Tyablikov, Dokl. Phys. 4, 589 (1959).

[9] F. Marsiglio, M. Schossmann, J.P. Carbotte, Phys. Rev. B 37, 4965 (1988).

[10] J.P. Carbotte, Rev. Mod. Phys 62, 1027 (1990).

[11] P. Morel, P.W. Anderson, Phys. Rev. 125, 1263 (1962).

[12] J. Bardeen, L.N. Cooper, J.R. Schrieffer Phys. Rev. 108, 1175 (1957). 